\title{
Response Characteristics of Lamb Trigeminal Neurons to Stimulation of the Oral Cavity and Epiglottis With Different Sensory Modalities
}

\author{
ROBERT D. SWEAZEY* AND ROBERT M. BRADLEY*† \\ *Department of Biologic and Materials Sciences, School of Dentistry \\ The University of Michigan, Ann Arbor, MI 48109 \\ +Department of Physiology, School of Medicine, The University of Michigan, Ann Arbor, MI 48109
}

Received 7 November 1988

\begin{abstract}
SWEAZEY, R. D. AND R. M. BRADLEY. Response characteristies of lamb trigeminal neurons to stimulation of the oral cavity and epiglottis with different sensory modalities. BRAIN RES BULL. 22(5) 883-891, 1989. - A region of the trigeminal complex located at the border of the subnucleus interpolaris and subnucleus caudalis receives not only trigeminal nerve inputs from the face, tongue and palate, but also afferent terminations from other nerves which innervate the oral cavity and upper airway. To increase our understanding of the types of sensory information relayed to this region of the trigeminal nucleus, we investigated the response characteristics of single neurons to stimulation of the tongue, palate and epiglottis. Receptive field size and location of 83 trigeminal neurons were mapped, and responses to mechanical, thermal and chemical stimuli were recorded. About $90 \%$ of the neurons had one receptive field and no convergence between the oral cavity and epiglottis was observed. Furthermore, only about $15 \%$ of the trigeminal neurons responded to more than one stimulus modality. A moving mechanical stimulus elicited responses in over $90 \%$ of the cells, and $84 \%$ responded to moving and punctate mechanical stimuli. These mechanosensitive neurons generally exhibited rapidly adapting responses. Thermal and chemical stimuli were relatively ineffective. Cooling a receptor surface most often produced excitation, and warming inhibition. Responses to chemical stimuli were only observed for salts at high concentrations. These results suggest that, like oral cavity information relayed by the trigeminal nerve, afferent terminations in the trigeminal nucleus from other nerves subserving the oral cavity and upper airway function to relay mechanical sensory information. This information may be important in the elicitation of mechanically evoked oral and upper airway reflexes, or may provide a pathway to the thalamus for qualitative and quantitative sensory information about these areas.
\end{abstract}

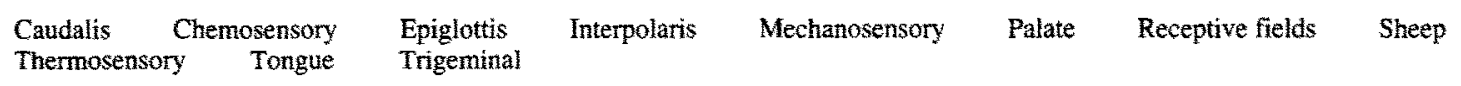

AFFERENT information from the oral cavity is represented in all divisions of the brainstem trigeminal complex $(1,29,45,46)$ and is conveyed by the various branches of the trigeminal nerve ( 29 , $45,46)$. Other cranial nerves which innervate the oral cavity and epiglottis terminate primarily in the nucleus of the solitary tract (NTS). However, there is a region in the caudal medulla encompassing the caudal subnucleus interpolaris and the rostral subnucleus caudalis which receives not only trigeminal inputs, but also a small number of afferent terminations from other cranial nerves which innervate the oral cavity and upper airway. These include terminations of the chorda tympani branch of the facial nerve $(36,54)$, the lingual-tonsillar branch of the glossopharyngeal nerve innervating the caudal tongue $(37,51)$, and the superior laryngeal nerve innervating the epiglottis $(14,38,51)$.

Numerous investigations have studied the response characteristics of neurons in the trigeminal complex to stimulation of the face, anterior oral cavity and tooth pulp $(15,41)$. In contrast, the responses of trigeminal neurons to stimulation of the caudal tongue and palate, and particularly the epiglottis, have rarely been investigated. Yet the information from these other receptor areas, in combination with that of more rostral oral regions, contribute important afferent input to the NTS for oral and upper airway reflex behaviors such as mastication, swallowing and coughing $(11,12,24,39)$. Therefore, afferent information from these receptive fields also might be important in any regulation of oral cavity and upper airway sensory reflexes by the trigeminal complex.

The present study was designed to examine the response characteristics of neurons in the restricted region of the trigeminal nucleus where afferent fibers from the oral cavity and upper airway both terminate. Single neuron responscs were investigated during stimulation of the oral cavity and epiglottis with different stimulus modalities to determine whether neurons received converging inputs, and to compare trigeminal neural response characteristics with previously recorded responses of NTS neurons which also receive information from these receptor areas. Lambs 
were used because the structure, number and distribution of taste buds in the oral cavity and epiglottis have been described (5, 31-33); there is anatomical information on the regions of the trigeminal nucleus which receive information from nontrigeminal oral and upper airway afferent nerves (51); and there are behavioral data on the reflex responses elicited by stimulation of these structures $(23,28)$. Furthermore, there is information on the response characteristics of NTS neurons to stimulation of the lamb epiglottis, tongue and palate $(52,53)$. Finally, the large size of the lamb oral cavity and epiglottis permits mechanical and thermal stimulation of restricted areas that would be very difficult in a smaller mammal,

\section{METHOD}

The methods used in the present study were similar to those reported in detail previously (53). Briefly, 43 Suffolk lambs (aged $30-80$ days) were anesthetized with sodium pentobarbital and the epiglotis and oral cavity exposed. The animal's head was placed in a stereotaxic frame and the surface of the brainstem exposed. The stereotaxic frame was then rotated laterally $45^{\circ}$ from horizontal to permit stimulation of the epiglottis, tongue and palate while recording from single trigeminal neurons.

Using the obex as a zero reference point, tungsten microelectrodes (2-3 M $\Omega$ impedance) were positioned over the trigeminal complex and advanced into the brain with a micromanipulator. The search for responsive trigeminal neurons was restricted to the caudal subnucleus interpolaris and rostral subnucleus caudalis from $2.0 \mathrm{~mm}$ rostral to $2.0 \mathrm{~mm}$ caudal to obex. This region of the trigeminal complex was chosen because the majority of trigeminal terminations of the lingual-tonsillar branch of the glossopharyngeal nerve which innervates the caudal oral cavity, and the superior laryngeal nerve which innervates the epiglottis, terminate in this area $(51)$.

Single trigeminal neurons were located either by stroking the receptor areas with a soft brush, or by applications of warm and cool rinse solutions, or a mixture of $1.0 \mathrm{M} \mathrm{NH}_{4} \mathrm{Cl}$ and $\mathrm{KCl}$. These stimuli have previously been shown to be effective for lamb and sheep chorda tympani, superior laryngeal and glossopharyngeal nerve fibers $(7,31,32)$ and NTS neurons $(6,52,53)$.

When a neuron was isolated, its receptive field was located using a glass probe or, if the neuron was not responsive to mechanical stimuli, the receptive field was defined using a thermal stimulator. Receptive fields were mapped onto representative drawings of the oral cavity or epiglottis. After determining a neuron's receptive field, responses to mechanical, thermal and chemical stimuli were recorded onto one channel of a tape recorder, and voice cues of experimental procedure were placed on a second channel.

Mechanical stimulation consisted of a moving glass probe (glass rod, $5 \mathrm{~mm}$ tip diameter), a moving soft brush, and punctate stimulation applied using a hand-beld, modified Grass strain gauge (circular stimulating surface, $5 \mathrm{~mm}$ in diameter). The strain gauge was connected to an amplifjer, chart recorder and third channel of a tape recorder to provide a permanent record of stimulation pressure. Punctate stimulation ranged from 1 to greater than 10 grams \pm 0.2 grams and was applied for 3 to $5 \mathrm{sec}$. All neurons received repeated applications of glass probe and brush stimulation and punctate stimulation was applied at least twice for $65 \%$ of the neurons. In addition, the responses to a moving stimulus were quantified for a subset of neurons ( $31 \%$ ) by moving the stimulator over the receptive field.

Thermal stimulation was accomplished by placing a watercirculating thermal probe (range $22-42^{\circ} \mathrm{C}$ ) on the receptor surface. $A$ thermistor on the end of the probe, connected to an amplifier. chart recorder and a fourth channel of the tape recorder, provided a permanent record of the temperature at the probe-mucosa boundary. The thermal stimulation sequence was repeated for all neurons which responded to thermal stimulation

Chemical stimuli applied to the tongue and palate were $1.0 \mathrm{M}$ $\mathrm{KCl} . \mathrm{NH}_{4} \mathrm{Cl}$ and $\mathrm{NaCl}$ and $0.01 \mathrm{~N} \mathrm{HCl}$ dissolved in distilled water. Distilled water also served as the rinse. Solutions applied to the epiglottis were $1.0 \mathrm{M} \mathrm{KCl}$ and $\mathrm{NH}_{4} \mathrm{Cl}, 0.01 \mathrm{~N} \mathrm{HCl}$ and distilled water. Water elicits a neural response in the superior laryngeal nerve and NTS when applied to the cpiglotis. Therefore, the chemicals which were applied to the cpiglottis were dissolved in $0.154 \mathrm{M} \mathrm{NaCl}$ which elicits minimal activity in superior laryngeal nerve fibers and NTS neurons during epiglottal stimulation (7.48. 52). This concentration of $\mathrm{NaCl}$ was also used as a rinse solution to remove chemical stimuli from the epiglottis. If a neuron responded to the $1.0 \mathrm{M}$ concentrations of salts it was subsequently tested for its response to 0.5 and $2.0 \mathrm{M}$ salts.

Syringes $(10 \mathrm{ml})$ were used to deliver $10 \mathrm{ml}$ of a stimulus solution to the receptor areas. Each chemical solution remained on the mucosa for a minimum of 10 sec and was then removed by 4 successive applications of $10 \mathrm{ml}$ of the appropriate rinse solution. Stimuli were delivered by hand at a flow rate of $10 \mathrm{ml} / 12 \mathrm{sec} \pm 1$ sec and all chemical and rinse solutions were delivered at oral cavity-epiglottis temperature $\left(33^{\circ} \mathrm{C}\right)$, Chemical stimuli for the oral cavity were applied in the following sequence: $\mathrm{NH}_{4} \mathrm{Cl}, \mathrm{KCl}, \mathrm{HCl}$, $\mathrm{NaCl}, \mathrm{NH}_{4} \mathrm{Cl}$. Stimuli for the epiglottis were applied as follows: $\mathrm{KCl}, \mathrm{NH}_{4} \mathrm{Cl}$, distilled water, $\mathrm{KCl}$. At least 2 min elapsed between stimulations to prevent cumulative adaptation (47), and for $90 \%$ of the neurons responsive to chemical stimuli, the total sequence of stimuli was repeated. As in previous investigations, the stability of the neural preparation was monitored by applying a chemical standard twice in the stimulation sequence (53). The chemical standard for neurons with oral cavity receptive fields was $\mathrm{NH}_{4} \mathrm{Cl}$, while $\mathrm{KCl}$ served as the standard for neurons with receptive fields on the epiglottis $(7,48,52,53)$.

After determining a neuron's response characteristics, the location of the recording site was marked by passing $35 \mu \mathrm{ADC}$ for 3-5 sec through the electrode. Following completion of the experiment the animal was given an overdose of pentobarbital and the brainstem removed and postfixed in $10 \%$ formalin. Coronal sections $(50 \mu \mathrm{m})$ were cut on a sliding microtome and mounted on slides coated with chromaltum. Sections were stained using the Auterta method (55) and recording sites were marked on standard drawings of the lamb brainstem.

Recorded neural impulses were converted into standard electrical impulses using a window discriminator. Intervals between pulses in milliseconds werc measured with a microcomputer (4). and this information was then converted to frequencies (impulses/ seck. In order to analyze only that portion of responses due to a stimulus, mean spontaneous frequency was stabtracted from evoked responses. The spontaneous frequency was calculated by averaging the $5 \mathrm{sec}$ immediately preceding a stimulation and subtracting this mean from the response evoked by that stimulation. For all neurons which received repeated presentations of the stimuli, the response was averaged across these repeated presentations.

A response to thermal stimulation was defined as an increase or decrease in the adjusted response frequency during any 5 sequential seconds of the stimulation period greater than the mean spontaneous activity \pm 2 S.D. and a neuron was considered responsive to a punctate or moving mechanical stimulus if the first $1 \mathrm{sec}$ adjusted response was greater than the mean spontaneous activity $=2$ S.D.

Because many neurons responded to mechanical stimulation, the mean response frequency due to the mechanical effects of flowing a chemical solution over the receptive field also was determined and subtracted from the responses elicited by chemical stimuli $(32,52)$. Previous investigations of lamb NTS neurons have shown that the responses to some chemical stimuli do not 


\section{TABLE}

MEAN RECORDING SITES (IN mm AND S.E.M.) FOR NEURONS ISOLATED IN LAMB TRIGEMINAL NUCLEUS

\begin{tabular}{llll}
\hline & Anterior-Posterior & Medial-Lateral & Dorsal-Ventral \\
\hline All Neurons & $0.22 \pm 0.07$ & $5.52 \pm 0.08$ & $2.81 \pm 0.05$ \\
Oral Cavity & $0.20 \pm 0.08$ & $5.45 \pm 0.09$ & $2.74 \pm 0.05$ \\
$\quad$ Palate & $0.18 \pm 0.11$ & $5.48 \pm 0.61$ & $3.0 \pm 0.10$ \\
Tongue & $0.15 \pm 0.14$ & $5.35 \pm 0.15$ & $2.65 \pm 0.06$ \\
$\quad$ T\&P & $0.52 \pm 0.29$ & $5.78 \pm 0.31$ & $2.69 \pm 0.14$ \\
Epiglottis & $0.35 \pm 0.16$ & $5.95 \pm 0.21$ & $2.74 \pm 0.13$ \\
\hline
\end{tabular}

attain peak frequency until some time following the onset of stimulation (52). Therefore, the presence of a response to chemical stimulation in trigminal neurons was defined as a difference in average adjusted response frequency (spontaneous frequency and response due to flow subtracted) during any 5 sequential seconds of the stimulation period greater than the mean \pm 2 S.D. of the spontaneous activity. This criterion permitted the analysis of responses which showed an increase or decrease in response frequency with time.

\section{RESULTS}

\section{Anatomical Organization}

Responses were recorded from 83 single trigeminal neurons; 31 neurons had a single receptive field on the ipsilateral tongue, 35 on the ipsilateral palate, 11 on the ipsilateral laryngeal surface of the epiglottis, and 6 neurons had two receptive fields, one on the tongue and one on the palate (TP neurons). Neurons were located in the dorsal one-third of the caudal subnucleus interpolaris or the rostral subnucleus caudalis. The oral cavity and epiglottis were somatotopically organized in an inverted medial-facing manner, with the oral cavity medial, the epiglottis lateral, and the palate ventral to the tongue. The recording sites of lamb trigeminal neurons, based on receptive field location, are shown in Fig. 1.

The mean recording sites of the different groups of neurons are given in Table 1. Comparison of recording sites among the different groups of trigeminal neurons revealed no significant differences in the anterior-posterior coordinates. However, as a group, neurons with oral cavity receptive fields were significantly more medial than neurons with receptive fields located on the epiglottis $(t=2.1, p<0.05$ ), and neurons with receptive fields on the palate were significantly more ventral than neurons with receptive fields on the tongue $(t=2.9, p<0.001)$.

\section{Receptive Fields}

The receptive fields of the 83 trigeminal neurons are mapped onto the schematic drawings of Fig. 2. Over $66 \%$ of the neurons had well defined receptive fields of less than $100 \mathrm{~mm}^{2}$; however, a few neurons had more diffuse receptive fields which covered over half of the ipsilateral tongue or palate. These very large receptive fields were usually composed of a region of high sensitivity surrounded by a large area in which stronger intensities of stimulation were required to elicit a response. Reflecting the difference in size between the oral cavity and epiglottis, receptive fields located on the epiglottis were generally much smaller than those observed in the oral cavity. Receptive field size ranged from a low of $8 \mathrm{~mm}^{2}$ on the epiglottis to a high of $700 \mathrm{~mm}^{2}$ on the tongue. The mean receptive field size for the different groups of neurons is presented in Table 2.

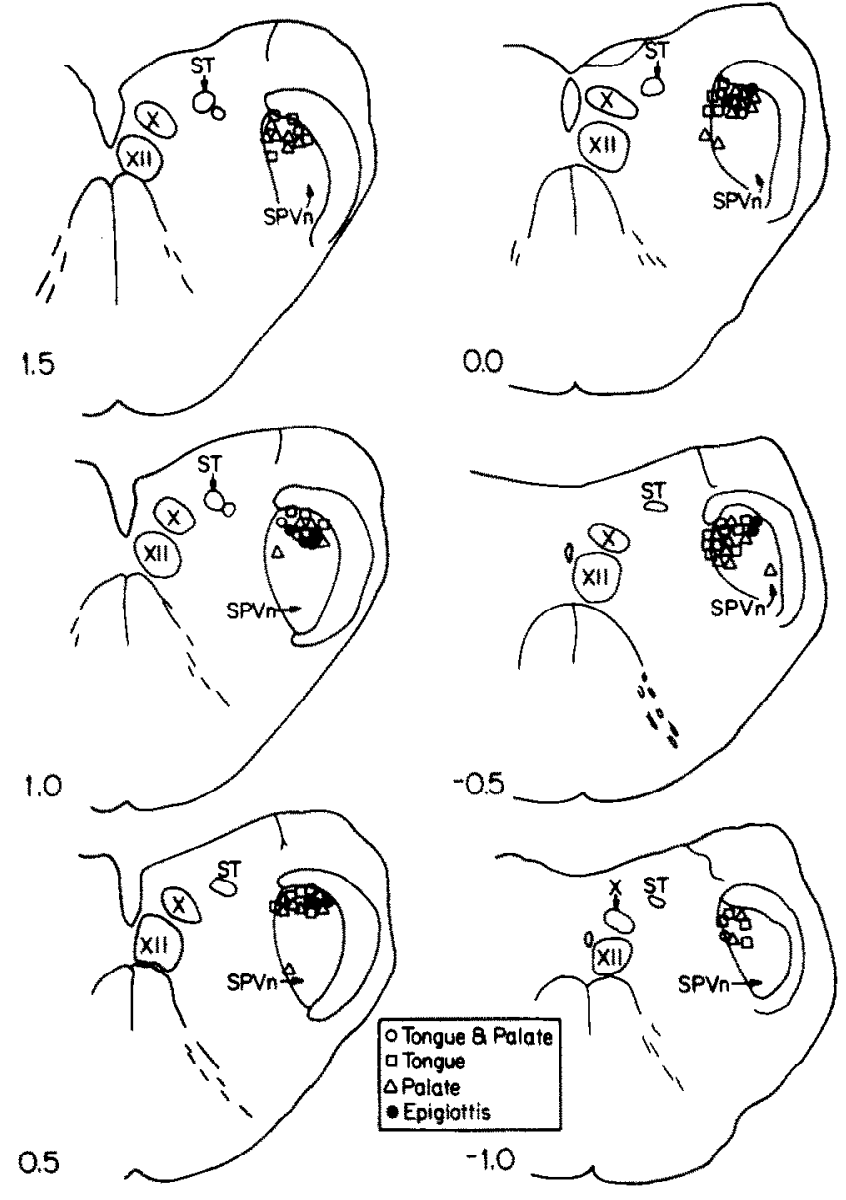

FIG. 1. Anatomical reconstructions of recording sites in the lamb trigeminal nucleus. The recording site of each isolated neuron is marked with a symbol (inset key) which represents the location of the neuron's receptive field. Note that neurons were generally located dorsal and dorsomedial in the trigeminal nucleus. Distances rostral or caudal to obex (in $\mathrm{mm}$ ) of each representative brainstem section are given at the lower left of each section. Abbreviations: SPVn, spinal trigeminal nucleus; ST, solitary nucleus; $X$, dorsal motor nucleus of the vagus; XII, hypoglossal nucleus.

The majority (5/6) of TP neurons had receptive fields located in the caudal oral cavity (Fig. 2, lower left). For all TP neurons, the receptive field on the palate was located directly above the receptive field on the tongue. Furthermore, the two receptive fields were usually about the same size, although 1 TP neuron had a receptive field on the palate which was four times larger than the receptive field on the tongue.

TABLE 2

MEAN RECEPTIVE FIELD SIZE (IN $\mathrm{mm}^{2}$ AND S.E.M.) FOR NEURONS ISOLATED IN LAMB TRIGEMINAL NUCLEUS

\begin{tabular}{lrr} 
All Neurons & $130.7 \pm 17.2$ \\
Palate & $132.3 \pm 28.0$ \\
Tongue & $143.9 \pm 28.7$ \\
T\&P-Palate & $214.7 \pm 100.9$ \\
T\&P-Tongue & $168.8 \pm 63.3$ \\
Epiglottis & $22.1 \pm 2.6$ \\
\hline
\end{tabular}


PALATE
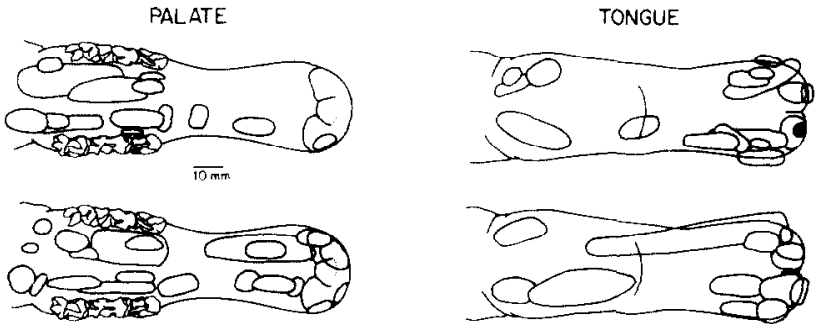

PALATE \& TONGUE

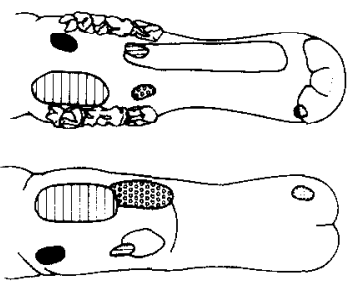

EPIGLOTTIS

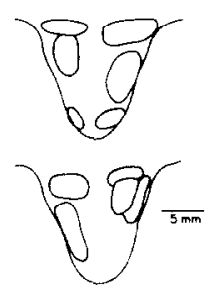

FIG. 2. Receptive fields of the 83 lamb trigeminal neurons. The receptive fields for the tongue (upper right), palate (upper left) and epiglottis (lower right) are from neurons which had only a single receptive field. For neurons responsive to stimulation of both the palate and tongue (lower left), the two receptive fields of an individual neuron are similarly shaded. All trigeminal neurons had receptive fields restricted to the ipsilateral tongue, palate or epiglottis. However, for clarity, receptive fields have been mapped onto both sides of the schematic drawings. The calibration bar in the upper left applies to the tongue and palate schematics, the one in the lower right to the drawings of the epiglottis.

\section{Response Characteristics}

The spontaneous rates of trigeminal neurons were usually low, ranging from 0 to $38 \mathrm{impulses} / \mathrm{sec}$. Eighteen neurons had no spontaneous activity and a majority of the remaining neurons had spontaneous rates of less than 5 impulses $/ \mathrm{sec}$. The mean spontaneous rate for all neurons was $2.5 \pm 0.62$ (S.E.M.) impulses $/ \mathrm{sec}$.

Eighty-eight percent of the neurons responded exclusively to one of the stimulus modalities, either mechanical (66 neurons) or thermal ( 7 neurons). The remaining neurons responded to 2 stimulus modalities ( 3 responded to both mechanical and thermal stimuli, and 7 exhibited a strong response to mechanical stimuli and a very weak response to chemical stimuli).

\section{Responses to Mechanical Stimuli}

Mechanical stimuli were the most effective of the three stimulus modalities regardless of receptive field location and most of the mechanosensitive neurons responded to both moving and punctate stimuli (see Figs. 3 and $4 \mathrm{~A}$ ). Over $80 \%$ of mechanosensitive neurons were rapidly adapting and many responded with a brief burst of activity to the onset and offset of a punctate stimulus. Furthermore, these rapidly adapting neurons usually had well defined receptive fields, but rapidly adapting neurons with large, diffuse receptive fields were occasionally observed. An example of a rapidly adapting neuron with an on-off response to punctate stimulation is shown in Fig. 3.

The remaining neurons responded to a punctate stimulus with either a sustained response or a response that was rapidly adapting to low stimulus intensities and slowly adapting to stronger pressures. The receptive fields of these neurons also were generally of moderate size and well defined. An example of a sustained
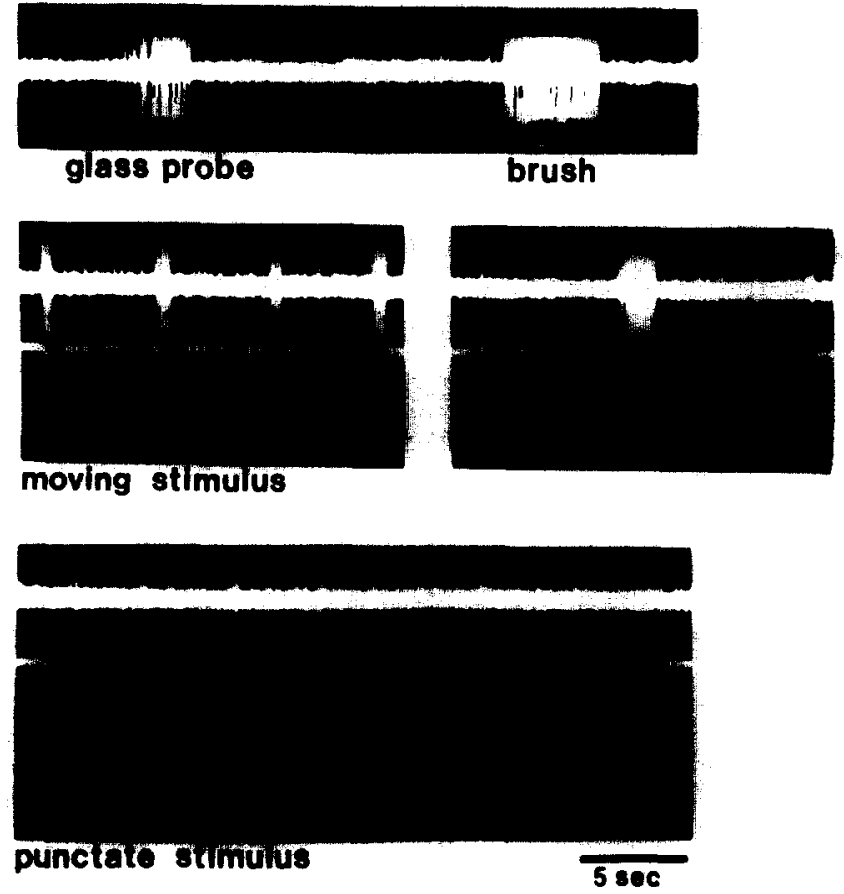

FIG. 3. Oscilloscope tracings of responses from a trigeminal neuron which had a receptive field on the caudal tongue. This neuron responded primarily to mechanical stimuli, but also exhibited a weak response to chemical stimuli (not shown). Responses to the different types of mechanical stimuli used in the study are presented in the different panels. Note that a moving stimulus (panel 2) produces a greater magnitide of response than a punctate stimulus and this neuron responded to the onset and offset of punctate stimuli. Periods of stimulation in the top panel are indicated by solid lines, periods of stimulation in the remaining panels are indicated by the stimulus record under the neural traces. Intensity of each stimulation is shown beneath the stimulus records. The time bar at the bottom right of the figure applics to all oscilloscope tracings except the one panel which contains its own time bar.

response to punctate stimuli is shown in Fig. 4A. Sustained responses to punctate stimuli were observed more frequently in neurons with epiglottal receptive fields $(27 \%)$ than in neurons having receptive fields in the oral cavity $(8 \%)$.

Although most neurons responded to both punctate and moving stimuli, $16 \%$ of mechanosensitive neurons with one receptive field did not respond to punctate stimuli. In addition, 3 of the $6 \mathrm{TP}$ neurons showed a mixed response in that they responded to punctate and moving stimuli on one of the receptive fields, but only to movement on the other.

Regardless of receptive field location, almost all neurons responded with increased frequency to increases in punctate stimulation intensity. Eighty-nine percent of the neurons responsive to punctate stimuli responded to all intensity levels from $1-10+$ grams. Stimulus intensities less than 1 gram were slightly less effective, eliciting responses in $70 \%$ of the punctate responsive cells. The mean response frequencies to punctate stimuli are shown in Fig. 5, which illustrates that an increase from 1 to 10 grams resulted in a six-fold increase in mean response frequency. The mean responses of neurons to punctate stimulation based on receptive field location are shown in Fig. 6. No significant differences existed between the slopes of the four response functions illustrated in Fig. 6. However, significant differences between the response magnitudes of the functions were observed, $F(3,63)=3.84, p<0.05$. Neurons with receptive fields on the 
A. Mechanical.
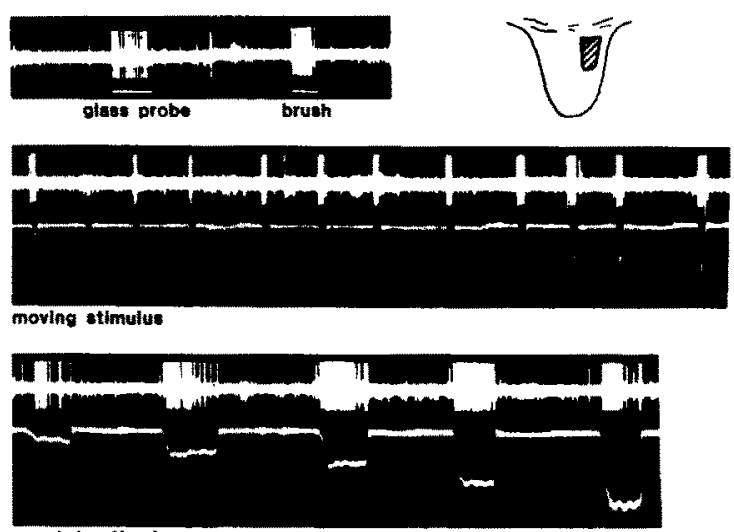

punctete otimulus

B. CHEMICAL

$\mathrm{KCl}$

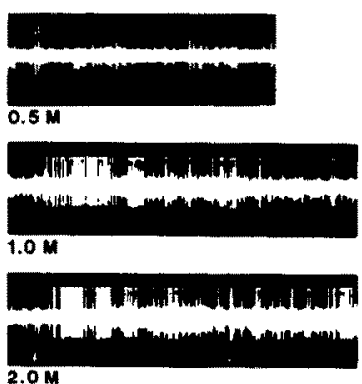

FIG. 4. Oscilloscope tracings of responses from a trigeminal neuron whose receptive field was located on the laryngeal surface of the epiglottis. This neuron responded to both mechanical and chemical stimuli.(A) Responses to different types of mechanical stimulation. (B) Responses to different concentrations of $\mathrm{KCl}$ and $\mathrm{NH}_{4} \mathrm{Cl}$. Note that this neuron did not respond to $0.5 \mathrm{M}$ concentrations of these salts and that the response to $\mathrm{NH}_{4} \mathrm{Cl}$ showed a slow increase. Onset of the chemical stimuli is denoted by the arrows at the bottom left of each set of tracings, onset of poststimulus rinses by the arrowheads in each panel. The location of this neuron's receptive field is shown in the schematic drawing at the upper right. The time bar applies to all tracings.

epiglottis, tongue or both the tongue and palate were significantly more responsive to punctate mechanical stimuli than neurons with palate receptive fields (pairwise comparisons, $p<0.05$ ). The relatively low responsiveness of neurons with palate receptive fields reflects the fact that a small number of neurons $(10 \%)$ did not increase their response frequency with increasing stimulus intensities. These neurons usually had receptive fields restricted to the palate, were rapidly adapting, and responded with only 1 or 2 impulses to a stimulus regardless of strength. Furthermore, these neurons always responded to stimulus strengths of less than 1.0 gram, and usually responded to the offset of the stimulus as well.

The glass probe and soft brush were the most effective stimuli. Examples of responses produced by these stimuli are shown in Figs. 3 and $4 \mathrm{~A}$. All mechanosensitive neurons responded to moving a glass probe over the receptive field and only one neuron failed to respond to brushing. Stimulation with a glass probe or brush also produced the largest mean response frequencies, $17 \pm 1.2$ and $14 \pm 1.2$ impulses/sec respectively.

Twenty-six of the neurons responsive to mechanical stimuli were investigated for their response to different intensities of a moving stimulus. Examples of the responses produced by moving

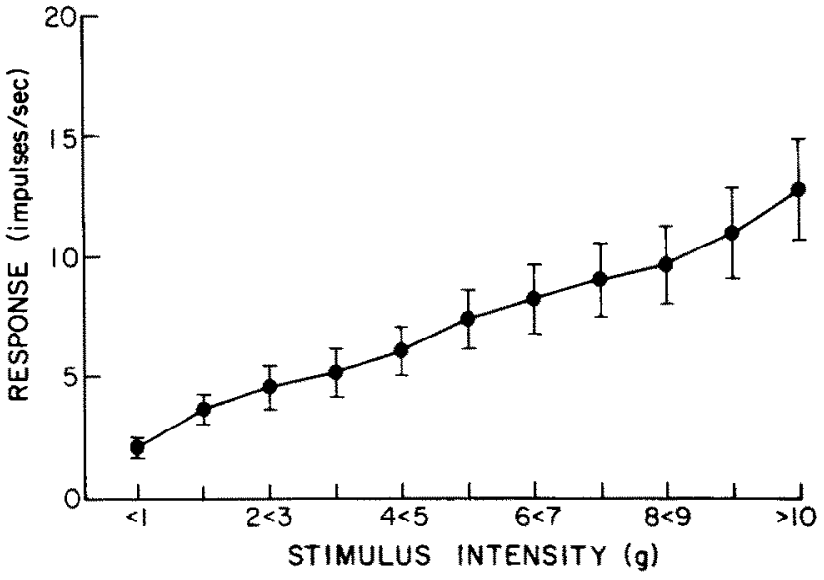

FIG. 5. Mean response frequencies and standard errors of 63 trigeminal neurons to different intensities of punctate mechanical stimulation. Responses to different stimulus intensities were grouped into the magnitude categories noted on the $\mathrm{x}$-axis. Note that the response magnitudes increase with increasing stimulus intensities.

the stimulator across the receptive field are shown in Figs. 3 and 4A. It can be seen in these figures that a moving stimulus not only produced a greater response frequency, but in most cases, smaller stimulus intensities were required to elicit a response. The mean response frequencies to increasing intensities of a moving stimulus are presented in Fig. 7. For comparison, the mean response frequencies of this subset of neurons to punctate stimuli also are displayed in this figure. It is obvious that moving the stimulator across the receptive field was much more effective than a punctate stimulus at all but the lowest level of stimulus intensity.

\section{Responses to Thermal Stimuli}

Thermal stimulation was less effective than mechanical for our sample of trigeminal neurons, with only 10 neurons responding to

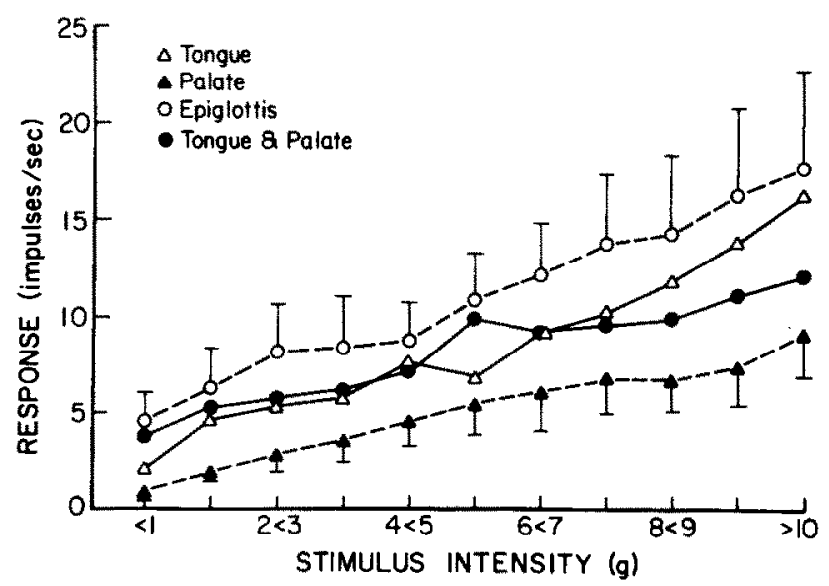

FIG. 6. Mean response frequencies of different groups of trigeminal neurons to different intensities of punctate mechanical stimulation. The neurons are grouped according to the location of their receptive field(s). The number of response functions per group were: tongue, 19; palate, 30; epiglottis, 10; tongue and palate, 9. Because of a large amount of overlap in the standard errors, only standard errors for the most (epiglottis) and least (palate) responsive groups of neurons are presented. 


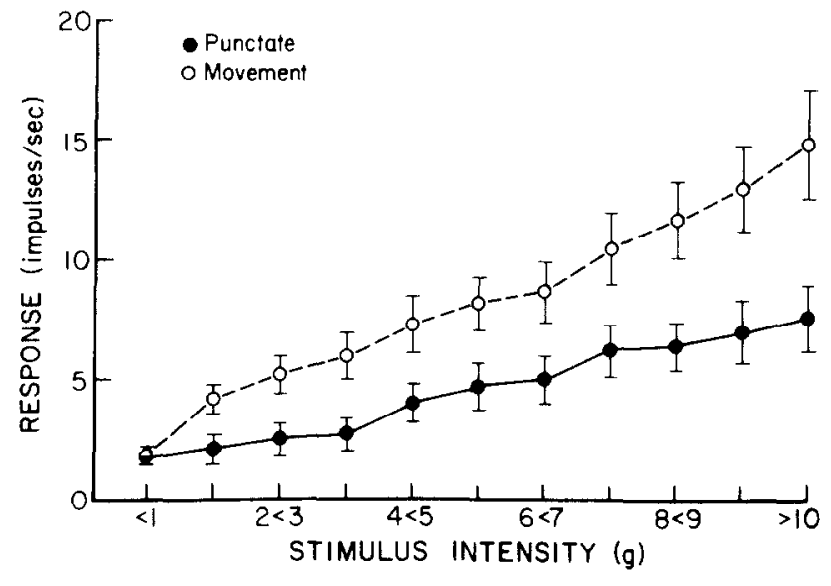

FIG. 7. Mean response frequencies and standard errors of 26 neurons in which responses to different intensities of both punctate and movement were determined. Responses to different stimulus intensities were grouped into the magnitude categories noted on the $\mathrm{x}$-axis. Note that moving the stimulator across the receptive field produced a larger response at all but the lowest level of stimulus intensity.

this stimulus modality. Seven of these neurons responded exclusively to thermal stimuli and hehaved in a manner similar to that reported for peripheral cold fibers. These neurons exhibited a static discharge (mean $=6.5 \pm 1.4$ impulses $/ \mathrm{sec}$ ), increased their activity when the receptive field was cooled, and responded to warming of the receptive field with a decrease in activity. The response to warming in these neurons usually reflected a decrease from ongoing rates of discharge to zero. Based on the first second of the response, cooling the receptive field in this group of thermal neurons produced an increase in activity which ranged from 11 to $35 \mathrm{impulses} / \mathrm{sec}$, while warming the receptive field resulted in a decrease ranging from 3 to $12 \mathrm{impulses} / \mathrm{sec}$. Receptive fields of thermosensitive neurons were located on the tongue, principally on the anterior one-third. Three of the 10 neurons which responded to thermal stimuli also responded to a mechanical stimulus. These neurons responded best to mechanical stimuli, exhibiting a slowly adapting response.

\section{Responses to Chemical Stimuli}

Chemical stimuli were the least effective of the stimulus modalities tested. Seven neurons exhibited a weak response to chemical stimuli and these responses were only observed to $1.0 \mathrm{M}$ or higher concentrations of $\mathrm{NH}_{4} \mathrm{Cl}$ or $\mathrm{KCl}$, or in the case of one neuron, $2.0 \mathrm{M} \mathrm{NaCl}$. None of the neurons responded to $\mathrm{HCl}$, and neurons with receptive fields on the epiglottis did not respond to water, normally an effective stimulus for this receptor area. Applications of $0.5 \mathrm{M}$ salts failed to elicit a response in any trigeminal neuron in which they were tested.

The receptive fields of the seven neurons responsive to chemical stimuli were restricted to either the tongue (5) or epiglottis (2). Only neurons with receptive fields on the tongue responded to 1.0 $\mathrm{M} \mathrm{NH}{ }_{4} \mathrm{Cl}$, while a $2.0 \mathrm{M}$ concentration of this salt produced a weak response in one of the neurons with an epiglottal receptive field. The adjusted mean responses to 1.0 and $2.0 \mathrm{M} \mathrm{NH}_{4} \mathrm{Cl}$ were $5.8 \pm 1.6(n=5)$ and $8.8 \pm 2.7$ impulses/sec $(n=6)$ respectively. $\mathrm{KCl}$ was even less effective than $\mathrm{NH}_{4} \mathrm{Cl}$ in its ability to elicit responses in trigeminal neurons. Only four of the neurons responded to $\mathrm{KCl}$ ( 2 tongue, 2 epiglottis). The adjusted mean response frequencies to 1.0 and $2.0 \mathrm{M} \mathrm{KCl}$ were $3.4 \pm 0.8$ and $5.3 \pm 0.9 \mathrm{impulses} / \mathrm{sec}$ respectively.
DISCUSSION

We recorded the responses of 83 neurons located in the dorsomedial areas of the caudal interpolaris and rostral caudalis, an area of the trigeminal complex which receives projections from the superior laryngeal nerve and glossopharyngeal nerve. Trigeminal neurons within this region usually responded to mechanical stimulation of receptor areas innervated by these two nerves and to stimulation of areas of the oral cavity innervated by different branches of the trigeminal nerve such as the lingual. The typical somatotopic organization was observed, with the mouth inverted and the anterior tongue medial and epiglottis lateral $(15,25,29$. 46). Over $90 \%$ of the neurons responded to at least one type of mechanical stimulation and a moving mechanical stimulus usually proved to be much more effective than a stationary stimulus.

The majority of neurons with oral cavity receptive fields were rapidly adapting, and many of these neurons responded to both the onset and offset of a punctate stimulus. Neurons with similar response characteristics have been reported in the peripheral nerves which innervate the oral cavity, in the trigeminal ganglion, and throughout the trigeminal complex of a large number of different species including cat, sheep and monkey $(1,3,10,15$. 19, 21 25,41$)$. Furthermore, investigations of peripheral nerves innervating the cat tongue (3), or cells in the subnucleus interpolaris (15) have reported that over $75 \%$ of neurons sampled showed rapidly adapting responses to mechanical stimuli. a percentage similar to that found in the present study.

We have demonstrated that neurons in caudal interpolaris and rostral caudalis respond to stimulation of the epiglottis. This result extends the findings of previous investigations which have shown that neurons in the principal and spinal trigeminal nucleus are influenced by electrical stimulation of the superior laryngeal nerve (17.44). Like neurons with oral cavity receptive fields, neurons with epiglottal receptive fields generally exhibited rapidly adapting responses to mechanical stimuli. However, neurons were more likely to exhibit slowly adapting responses if they had a receptive field on the epiglottis than if they had a receptive field in the oral cavity. Studies of the superior laryngeal nerve in cat and rabbit note that a number of fibers are of the slowly adapting type. although there is some disagreement as to the proportion of slowly to rapidly adapting responses in this nerve $(10,42)$. Our results suggest that the majority of mechanical information relayed to the trigeminal nucleus from the epiglottis concerns the onset and offset of a stimulus. Nevertheless, like fibers in the superior laryngeal nerve, a relatively large percentage of neurons in the trigeminal nucleus do continue to respond for as long as the stimulus is present on the epiglottis.

The most frequently observed response characteristics and receptive field properties of rapidly adapting trigeminal neurons were similar to fast-adapting type I fibers described by Knibestöl $(26,27)$. These rapidly-adapting trigeminal neurons exhibited responses restricted to the transient phases of the stimulus and had relatively well defined, albeit larger receptive fields than peripheral fast-adapting type I fibers. Trigeminal neurons with slowlyadapting responses had response characteristics that were very similar to Knibestöl's slowly-adapting type I units in that they responded during the static phase of stimulation, often showed a greater sensitivity to the transient phase of stimulation, and had quite well defined receptive fields (Fig. 4).

The small number of neurons which required a strong punctate stimulus probably reflect an input from submucosal mechanoreceptors. For example, there are reports of peripheral nerve responses to stimulation of the sheep's tongue which required strong pressure or distortion of the surface of the tongue (19), suggesting that submucosal receptors in the tongue musculature were being stimulated. It also is possible that these high threshold neurons are involved in nociception. Amano et al. (1) reported that 
some nociceptive neurons in the cat trigeminal nucleus had thresholds between 5-14 grams which is similar to the intensity required to activate the small numbers of high threshold trigeminal neurons in our investigation.

The majority of trigeminal neurons we sampled responded better to a moving mechanical stimulus, or solely to moving stimuli. Similar responses to mechanical stimuli have been observed in peripheral nerves innervating the mucosa of the cat oral cavity and upper airway, or the human glabrous skin $(3,22,49)$. For example, in an investigation of the mechanosensitivity of the cat anterior tongue, Biedenbach and Chan (3) reported that rapidly adapting fibers in the chorda tympani and lingual nerve could be classified into two groups. The first group responded to light mechanical stimulation and had a higher discharge to moving stimuli. This is similar to what was observed for the majority of neurons in the present study (see Fig. 7). A second group of rapidly adapting neurons exhibited responses only to movement across the receptive field, a situation similar to that observed for $16 \%$ of the mechanosensitive trigeminal neurons which were sampled. Furthermore, Biedenbach and Chan found that this second group (movement-only) of neurons comprised the principal mechanosensory response in the chorda tympani nerve, which sends a small projection to the region of the trigeminal nucleus investigated in the present study (54). These data suggest that many trigeminal neurons which respond to movement may receive inputs from a specific group of afferent fibers and that the response properties observed in the periphery are maintained across the synapse. Moreover, for neurons with rostral tongue receptive fields this input may originate not only from the lingual branch of the trigeminal nerve, but also possibly from the small number of chorda tympani nerve fibers which terminate in the trigeminal nucleus.

Although inputs from certain groups of afferent fibers may account for the better response to movement in some neurons, it is also likely that moving the stimulator over the receptive field produced a greater response because more receptors were stimulated. This is because the head of the stimulator was usually smaller than the receptive field. Therefore, only by moving the stimulator across the receptive field would all receptors be stimulated.

Using very similar stimuli, we have previously determined the functional characteristics of neurons in a region of the NTS involved in reflex responses to stimulation of the oral cavity and epiglottis (53). A comparison of the responses to mechanical stimuli between trigeminal neurons with receptive fields in the oral cavity or epiglottis, and those NTS neurons with comparable receptive fields indicates similarities in these two nuclei. Both populations of neurons responded with increased response frequency to increases in magnitude of a mechanical stimulus, and both populations responded better to a moving than a punctate stimulus. Furthermore, the response magnitudes produced by different intensities of mechanical stimulation were quite similar.

Responses to thermal stimuli were observed only in a few of the trigeminal neurons that we sampled. Neurons which responded exclusively to temperature exhibited response characteristics similar to those described for "cold units" in peripheral nerves, including those which innervate the oral cavity, face and upper airway $(2,19,42)$. Neurons with response characteristics similar to those in the present study also have been observed in trigeminal nuclei of other species $(40,41)$. The fact that most thermosensitive neurons had receptive fields on the anterior tongue suggests that these neurons receive temperature information from the lingual nerve which contains a number of cold fibers $(16,19,57)$.

Chemical stimuli usually did not elicit responses in our sample of trigeminal neurons. Very strong concentrations $(1.0 \mathrm{M}$ or larger) of salts were required to elicit weak responses and concentrations of salts $(0.5 \mathrm{M})$ or acids $(0.01 \mathrm{~N})$ capable of eliciting strong, sustained responses in peripheral gustatory nerves and central gustatory nuclei never elicited responses in trigeminal neurons. This suggests that these chemical stimuli were not acting on the gustatory receptors, but instead might be acting nonspecifically on mechanoreceptors, or perhaps activating nociceptive fibers in the tongue and epiglottis.

The rare occurrence of chemical responses in trigeminal neurons is an important difference between the trigeminal nucleus and the NTS. The NTS is the principal site in the brainstem for termination of all gustatory afferents, whereas the trigeminal nucleus is generally acknowledged to be involved in processing mechanical, thermal and nociceptive information. Thus it is not surprising that chemical concentrations which produce robust responses in the NTS $(0.5 \mathrm{M}$ salts and $0.01 \mathrm{~N} \mathrm{HCl})(6,52,53) \mathrm{did}$ not elicit responses in trigeminal neurons. In our previous investigations of the reflexogenic regions of the NTS $(52,53)$, we found that NTS neurons responded to chemical stimuli, especially when applied to the lingual surface of the epiglottis, suggesting that the NTS was involved in upper airway reflexes which are elicited by chemical stimuli $(23,24,28)$. The results of the present investigation indicate that neurons in regions of the trigeminal nucleus we investigated do not participate in the reflex behaviors which are elicited by applications of chemical stimuli to the epiglottis or oral cavity.

Only a small number of trigeminal neurons received input from more than one receptive field. This finding is similar to that reported for the cat subnucleus interpolaris and caudalis $(1,15$, 17). However, similar to previous investigations of the trigeminal nucleus in other species, convergence of primary afferent fibers was reflected in the size of the lamb trigeminal neuron receptive fields. The receptive fields of trigeminal neurons which received oral cavity inputs were generally much larger than those reported for primary afferent fibers $(19,41)$, and were similar to receptive field sizes reported for cat oralis and caudalis neurons $(1,18)$. The receptive field sizes of trigeminal neurons with epiglottal receptive fields also were larger than has been reported for superior laryngeal nerve fibers (10).

The lack of convergence of tongue, palate and epiglottal afferent fibers onto neurons in the trigeminal nucleus is a result similar to that previously observed for NTS neurons (53). In both studies, neurons usually responded to stimulation of just one of the three areas stimulated, although neurons which received information from both the tongue and palate were found in larger numbers in the NTS.

In our previous investigations of the NTS, most neurons responded to more than one stimulus modality $(52,53)$. In contrast to the multimodal nature of NTS neurons, trigeminal neurons which receive oral cavity or epiglottal inputs rarely responded to more than one stimulus modality. Eighty-eight percent of trigeminal neurons responded to just one stimulus modality, whereas over $68 \%$ of NTS neurons were multimodal. This difference would be even more pronounced if trigeminal neurons' responses to chemical stimuli had been classified by their responses to $0.5 \mathrm{M}$ salts as was done for NTS neurons. Since trigeminal neurons did not respond to $0.5 \mathrm{M}$ salts, less than $4 \%$ of the trigeminal neurons would have been classified as multimodal. These data indicate that unlike neurons in the NTS, cells in regions of the trigeminal nucleus we sampled do not have a role in integrating sensory information from different modalities.

Trigeminal neurons responded to stimulation of the oral cavity and upper airway in a manner that would preserve the nature of the peripheral stimulus. Therefore, they appear to function as a relay station whose primary function is to convey information, particularly mechanosensory information, to other neuronal populations. Much of this information probably is relayed to brainstem sensory 
and motor nuclei involved in oral and upper airway reflexes which can be elicited by mechanical stimuli. Such reflexes include tongue position, mastication and swallowing $(8,13,35,50,56)$. However, these trigeminal neurons also may provide an important pathway by which quantitative and qualitative mechanosensory information reaches the thalamus. Anatomical and physiological studies in a number of different species have found that interpolaris and caudalis both send projections to the thalamus $(9,15,17$. 30 ). On the other hand, although much more afferent information from the caudal tongue, epiglottis and other upper airway struc- tures terminates in areas of the NTS involved in reflex function. cvidence suggests that these NTS areas do not project to more rostral brain structures $(20,34,43)$. Thus, information relayed to the thalamus and eventually to the cerebral cortex via the trigeminal complex may be responsible for much of the "touch" sensation associated with the upper airway and oral cavity.

\section{ACKNOWLEDGEMENT}

The work supported by NIH Grant DE05728 to R.M.B.

\section{REFERENCES}

1. Amano, N.; Hu, J. W.; Sessle, B. J. Responses of neurons in feline trigeminal subnucleus caudalis (medullary dorsal horn) to cutaneous intraoral and muscle afferent stimuli. J. Neurophysiol. 55:227-243; 1986.

2. Benzing, H.; Hensel, H.; Wurster, R. Integrated static activity of lingual cold receptors. Pflugers Arch. 311:50-54; 1969.

3. Biedenbach, M. A.; Chan, K. Y. Tongue mechanoreceptors: comparison of afferent fibers in the lingual nerve and chorda tympani. Brain Res. 35:584-588; 1971.

4. Bradley. R. M. Tapping into the brain. Microcomputing 62:72-79: 1982.

5. Bradley, R. M.; Cheal, M. L.; Kim, Y. H. Quantitative analysis of developing epiglottal taste buds in sheep. J. Anat. 130:25-32; 1980.

6. Bradley, R. M.; Mistretta, C. M. Developmental changes in neurophysiological taste responses from the medulla in sheep. Brain Res. 191:21-34; 1980

7. Bradley, R. M.; Stedman, H. M.; Mistretta, C. M. Superior laryngeal nerve response patterns to chemical stimulation of sheep epiglottis. Brain Res. 276:81-93; 1983

8. Bratzlavsky, M. Reflexes with intraoral afferents in human lip musculature. Exp. Neurol. 37:179-187; 1972.

9. Burton, H.; Craig, A. D. Distribution of trigeminothalamic projection cells in cat and monkey. Brain Res. 161:515-521; 1979.

10. Davis, P. J.; Nail, B. S. The sensitivity of laryngeal epithelial receptors to static and dynamic forms of mechanical stimulation. In: Fujimura, O., ed. Vocal physiology: Voice production, mechanisms and functions. New York: Raven Press, Ltd.; 1988:1-18.

11. Doty, R. W. Neural organization of deglutition. In: Code, C. F., ed. Handbook of physiology, sect. 6 alimentary canal, vol. 4. Washington, DC: American Physiological Society; 1968:1861-1902

12. Dubner, R.; Sessle, B. J.; Storey, A. The neural basis of oral and facial function. New York: Plenum Press; 1978.

13. Gottschaldt, K.-M.; Young, D. W. Quantitative aspects of responses in trigeminal relay neurones and interneurones following mechanical stimulation of sinus hairs and skin in the cat. J. Physiol. 272:85-103; 1977.

14. Hanamori, T.: Smith, D. V. Central projections of the hamster superior laryngeal nerve. Brain Res. Bull. 16:271-279; 1986

15. Hayashi, H.; Sumino, S.; Sessle, B. J. Functional organization of trigeminal subnucleus interpolaris: Nociceptive and innocuous afferent inputs, projections to thalamus, cerebellum, and spinal cord, and descending modulation from periaqueductal gray. J. Neurophysiol. $51: 890-905 ; 1984$

16. Hensel, H.; Zotterman, Y. The response of the cold receptors to constant cooling. Acta Physiol. Scand. 22:96-105; 1951

17. Hu, J. W.; Dostrovsky, J. O.; Sessle, B. J. Functional properties of neurons in cat trigeminal subnucleus caudalis (medullary dorsal horn). I. Responses to oral-facial noxious and nonnoxious stimuli and projections to thalamus and subnucleus oralis. J. Neurophysiol. 45:173-192; 1981

18. Hu, J. W.; Sessie, B. J. Comparison of responses of cutaneous nociceptive and nonnociceptive brain stem neurons in trigeminal subnucleus caudalis (medullary dorsal horn) and subnucleus oralis to natural and electrical stimulation of tooth pulp. J. Neurophysiol. $52: 39-53 ; 1984$

19. Iggo, A.; Leek, B. F. The afferent innervation of the tongue of the sheep. In: Hayashi, T., ed. Olfaction and taste II. Oxford: Pergamon Press; 1967:493-507.
20. Jean, A.; Car, A.; Roman, C. Comparison of activity in pontine versus medullary neurones during swallowing. Exp. Brain. Res. 22:211-220; 1975 .

21. Johansson, R. S.; Trulsson, M.; Olsson, K. A.; Westberg, K.-G. Mechanoreceptor activity from the human face and oral mucosa. Exp. Brain Res. 72:204-208; 1988.

22. Johansson, R. S.; Vallbo, A. B. Tactile sensory coding in the glabrous skin of the human hand. Trends Neurosci. 6:27-32;1983.

23. Johnson, P.; Robinson, J. S.; Salisbury, D. The onset and control of breathing after birth. In: Comline, K. S ; Cross, K. W : Dawes, G. S.: Nathanielsz, P. W., eds. Foetal and neonatal physiology. Cambridge: Cambridge University Press; 1973:217-221.

24. Johnson, P.; Salisbury, D. M.; Storey, A. T. Apnoea induced by stimulation of sensory receptors in the larynx. In: Bosma, J. F.; Showacre, J., eds. Development of upper respiratory anatomy and function: Implications for sudden infant death syndrome. Washington, DC: US Government Printing Office; 1975:160-183.

25. Kerr, F. W. L.; Kruger, L.; Schwassmann, H. O.; Stern, R. Organization of mechanoreceptor units in the trigeminal nuclear complex of the macaque. J. Comp. Neurol. 134:127-144; 1968.

26. Knibestöl, M. Stimulus-response functions of rapidly adapting mechanoreceptors in the human glabrous skin area. J. Physiol. 232: $427-452 ; 1973$.

27. Knibestöl, M. Stimulus-response functions of slowly adapting mechanoreceptors in the human glabrous skin area. J. Physiol. 245:63-80; 1975

28. Kovar, I.; Selstam, U.; Catterton, W. Z.; Stahlman, M. T.; Sundell, H. W. Laryngeal chemoreflex in newborn lambs: respiratory and swallowing response to salts, acids, and sugars. Pediatr. Res. 13: $1144-1149 ; 1979$.

29. Kruger, L.; Michel, F. A single neuron analysis of buccal cavity representation in the sensory trigeminal complex of the cat. Arch. Oral Biol. 7:491-503; 1962.

30. Matsushita, M.; Ikeda, M.; Okado, N. The cells of origin of the trigeminothalamic, trigeminospinal and trigeminocerebellar projections in the cat. Neuroscience $7: 1439-1454 ; 1982$.

31. Mistretta, C. M.; Bradley, R. M. Neural basis of developing salt taste sensation: response changes in fetal, postnatal, and adult sheep. $J$. Comp. Neurol. 215:199-210; 1983.

32. Mistretta, C. M.; Bradley, R. M. Developmental changes in taste responses from glossopharyngeal nerve in sheep and comparisons with chorda tympani responses. Dev. Brain Res. 11:107-117; 1983.

33. Mistretta, C. M.; Gurkan, S.; Bradley, R. M. Morphology of chorda tympani fiber receptive fields and proposed neural rearrangements during development. J. Neurosci. 8:73-78; 1988 .

34. Morest, D. K. Experimental study of the projections of the nucleus of the tractus solitarius and the area postrema in the cat. J. Comp. Neurol. 130:277-300; 1967.

35. Morimoto, T.; Takata, M.; Kawamura, Y. Effect of lingual nerve stimulation on hypoglossal motoneurons. Exp. Neurol. 22:174-190; 1968.

36. Nomura, S.; Mizuno, N. Central distribution of afferent and efferent components of the chorda tympani in the cat as revealed by the horseradish peroxidase method. Brain Res. 214:229-237; 1981.

37. Nomura, S.; Mizuno, N. Central distribution of afferent and efferent components of the glossopharyngeal nerve: an HRP study in the cat. Brain Res. 236:1-13; 1982.

38. Nomura, S.; Mizuno, N. Central distribution of efferent and afferent 
components of the cervical branches of the vagus nerve. A HRP study in the cat. Anat. Embryol. 166:1-18; 1983.

39. Pommerenke, W. T. A study of the sensory areas eliciting the swallowing reflex. Am. J. Physiol. 84:36-41; 1928.

40. Poulos. D. A. Central processing of cutaneous temperature information. Fed. Proc. 40:2825-2829; 1981.

41. Rowe, M. J.; Sessle, B. J. Responses of trigeminal ganglion and brainstem neurones in the cat to mechanical and thermal stimulation of the face. Brain Res. 42:367-384; 1972.

42. Sampson, S.; Eyzaguirre, C. Some functional characteristics of mechano-receptors in the larynx of the cat. J. Neurophysiol. 27: $464-480 ; 1964$.

43. Sessle, B. J. Excitatory and inhibitory inputs to single neurones in the solitary tract nucleus and adjacent reticular formation. Brain Res. $53: 319-331 ; 1973$.

44. Sessle, B. J.; Greenwood, L. F. Inputs to trigeminal brain stem neurons from facial, oral, tooth pulp and pharyngolaryngeal tissues: I. Responses to innocuous and noxious stimuli. Brain Res. 117:211226; 1976.

45. Shigenaga, Y.; Chen, I. C.; Suemune, S.; Nishimori, T.; Nasution, I. D.; Yoshida, A.; Sato, H.; Okamoto, T.; Sera, M.; Hosoi, M. Oral and facial representation within the medullary and upper cervical dorsal horns in the cat. J. Comp. Neurol. 243:388-408; 1986.

46. Shigenaga, Y.; Okamoto, T.; Nishimori, T.; Suemune, S.; Nasution, I. D.; Chen, I. C.; Tsuru, K.; Yoshida, A.; Tabuchi, K.; Hosoi, M.; Tsuru, $\mathrm{H}$. Oral and facial representation in the trigeminal principal and rostral spinal nuclei of the cat. J. Comp. Neurol. 244:1-18; 1986.

47. Smith, D. V.; Bealer, S. L.; Van Buskirk, R. L. Adaptation and recovery of the rat chorda tympani response to $\mathrm{NaCl}$. Physiol. Behav $20: 629-636 ; 1978$.

48. Stedman, H. M.; Bradley, R. M.; Mistretta, C. M.; Bradley, B. E. Chemosensitive responses from the cat epiglottis. Chem. Senses $5: 233-245 ; 1980$.

49. Storey, A. T. A functional analysis of sensory units innervating epiglottis and larynx. Exp. Neurol. 20:366-383; 1968.

50. Storey, A. T. Laryngeal initiation of swallowing. Exp. Neurol. 20:359-365; 1968.

51. Sweazey, R. D.; Bradley, R. M. Central connections of the lingualtonsillar branch of the glossopharyngeal nerve and the superior laryngeal nerve in lamb. J. Comp. Neurol. 245:471-482; 1986.

52. Sweazey, R. D.; Bradley, R. M. Response of lamb nucleus of the solitary tract neurons to chemical stimulation of the epiglottis. Brain Res. 439:195-210; 1988.

53. Sweazey, R. D.; Bradley, R. M. Responses of neurons in the lamb nucleus tractus solitarius to stimulation of the caudal oral cavity and epiglottis with different stimulus modalities. Brain Res. 480:133-150; 1989.

54. Whitehead, M. C.; Frank, M. E. Anatomy of the gustatory system in the hamster: central projections of the chorda tympani and the lingual nerve. J. Comp. Neurol. 220:378-395; 1983.

55. Wolf, G. Elementary histology for neuropsychologists. In: Meyers, D. D.. ed. Methods in psychobiology, vol. 1. London: Academic Press; 1971:283-300.

56. Yamamoto, T. Linguo-hypoglossal reflex: effects of mechanical thermal and taste stimuli. Brain Res. 92:499-504; 1975.

57. Zotterman, Y. Specific action potentials in the lingual nerve of cat. Skand. Arch. Physiol. 75:106-119; 1936. 